\title{
ALL-OPTICAL NONLINEAR SIGNAL PROCESSING AT A RZ RECEIVER
}

\author{
Sonia Boscolo, Sergei K. Turitsyn, and Keith J. Blow \\ Photonics Research Group, School of Engineering and Applied Science, \\ Aston University, Birmingham B4 7ET, United Kingdom
}

\begin{abstract}
We propose a novel all-optical signal processor for use at a return-to-zero receiver utilising loop mirror intensity filtering and nonlinear pulse broadening in normal dispersion fibre. The device offers reamplification and cleaning up of the optical signals, and phase margin improvement. The efficiency of the technique is demonstrated by application to $40 \mathrm{Gbit} / \mathrm{s}$ data transmission.
\end{abstract}

\section{INTRODUCTION}

In optical fibre communication systems using return-to-zero (RZ) modulation format, an electrical low-pass filter is normally employed after direct detection at the optical receiver. This filter limits the receiver bandwidth in order to reduce the noise power mainly due to accumulated amplified spontaneous emission noise generated by amplifier repeaters. Also, the expansion of the pulse width given by the filter reduces the influence of the fluctuation of pulse position in time caused by timing jitter [1]. But there exists a trade-off in the cut-off frequency of the filter among inter-symbol interference, signal amplitude, and signal-to-noise ratio (SNR). In this paper, we present a novel scheme of all-optical nonlinear signal processing for application to a RZ optical receiver front-end, which combines the intensity filtering action of a nonlinear optical loop mirror (NOLM) for reamplification and cleaning up of the optical signals $[2,3]$ with the nonlinear pulse broadening in a normal dispersion fibre (NDF) for phase margin improvement $[4,5]$. The efficiency of the proposed technique is numerically demonstrated by application to $40 \mathrm{Gbit} / \mathrm{s} \mathrm{RZ}$ data transmission. 


\section{OPERATION PRINCIPLE AND CONFIGURATION}

The proposed pulse processor consists of an optical amplifier, a section of NDF, and an unbalanced NOLM (see Fig. 1). Qualitatively, the idea for the method is as follows. An input pulse to the pulse processor is amplified to the preferred power level of the device by the optical amplifier. During transmission along the NDF, the temporal waveform of the pulse is changed to a rectangular-like profile by the combined action of group-velocity dispersion and Kerr nonlinearity [6]. As a result, the pulse width is broadened and the centre portion of the pulse is changed to be flat. By utilising this property, the phase margin of a RZ pulse train can be improved $[4,5]$. The phase margin improvement enables reduction of the influence of the displacement of pulse position at the receiver caused by timing jitter. Following the NDF, the pulse enters the NOLM. The unbalanced NOLM acts as a saturable absorber [7] and, hence, filters out low-intensity noise and dispersive waves from the higherpower pulse. This allows for restoration of the pulse amplitude and cleaning up of the distorted pulse. Also, whenever the NOLM operates in the region just after the peak of its switching curve, it enables stabilisation of amplitude fluctuations. In the case of a pulse train, the noise and radiative background in the zero timing slots is suppressed by the saturable absorption action of the NOLM, and the amplitude jitter of ones is also reduced $[2,3]$.

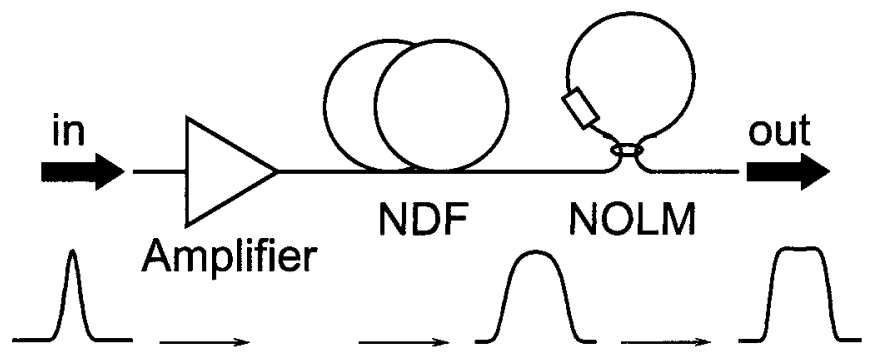

Figure 1. Schematic diagram of the pulse processor.

In the sample system used for demonstration of the technique, the amplifier is an erbium-doped fibre amplifier (EDFA) with a noise figure of $4.5 \mathrm{~dB}$. The NDF has a dispersion of $-20 \mathrm{ps} /(\mathrm{nm} \mathrm{km})$, an effective area of $30 \mu \mathrm{m}^{2}$, and an attenuation of $0.24 \mathrm{~dB} / \mathrm{km}$. The NOLM incorporates a 50:50 coupler, and a $1.5 \mathrm{~km}$-long loop of dispersion-shifted fibre with zero dispersion, an effective area of $25 \mu \mathrm{m}^{2}$, and an attenuation of $0.3 \mathrm{~dB} / \mathrm{km}$. Unbalancing of the NOLM is achieved with an optical attenuator asymmetrically placed in the loop, and the loss of the loop attenuator is $-27.1 \mathrm{~dB}$. The NOLM is preferably operated in the stable region of its switching curve. 


\section{MODELLING RESULTS}

To demonstrate the efficiency of the proposed pulse processor, without loss of generality, the following model simulations are run. $2^{7}-1$ pseudorandom RZ single-channel pulse trains are transmitted at $40 \mathrm{Gbit} / \mathrm{s}$ in a dispersionmanaged system whose transmission performance is severely degraded by intrachannel nonlinear effects when regenerators are not used (see [8] for details). In such a system, the periodical deployment of in-line NOLMs effectively stabilises the accumulation of amplitude noise and pulse distortion mainly driven by the intra-channel four-wave mixing, and the transmission distance is limited by intra-channel cross-phase modulation-induced timing jitter [8]. This presents a good model situation to demonstrate the action of the proposed device. The pulses after $20000 \mathrm{~km}$ transmission are used as the input for the pulse processor. The input full-width at half-maximum (FWHM) pulse width and energy are approximately $7 \mathrm{ps}$ and $0.011 \mathrm{pJ}$, respectively. The signal quality is evaluated in terms of the standard (Gaussian-based) $Q$-factor.
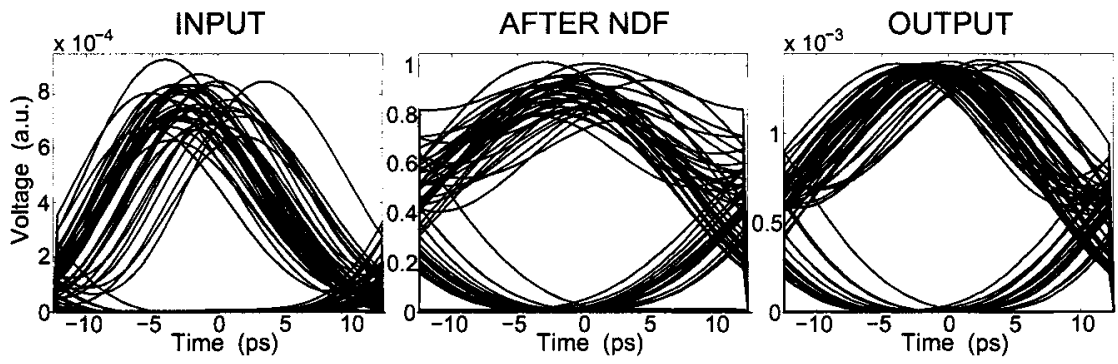

Figure 2. Eye-diagrams in the pulse processor.

Figure 2 shows an example of signal eye-diagrams at the pulse processor input, the NDF output, and the pulse processor output. In this example, the gain of the EDFA is $33.5 \mathrm{~dB}$, the length of the NDF is $0.5 \mathrm{~km}$, and a fifthorder Bessel filter with a cut-off frequency of $30 \mathrm{GHz}$ is used as a receiver low-pass filter. It can be seen that the input eye is closed mainly due to a significant timing jitter of the optical pulses. Dispersion and nonlinearity in the NDF broaden the pulse duration and simultaneously flatten the pulse shape. In this example, the FWHM pulse width is broadened to approximately 25 ps. Consequently, the eye opening at the NDF output is wider than at the pulse processor input. It is also seen that the amplitude jitter of pulses at the centre of the bit slot is slightly smaller. The additional widening of eye opening that can be observed at the pulse processor output is given by a significant reduction of the amplitude jitter provided by the NOLM.

The improvement of the signal quality that can be achieved in the nonlinear pulse processor-modified receiver with respect to the conventional receiver is 

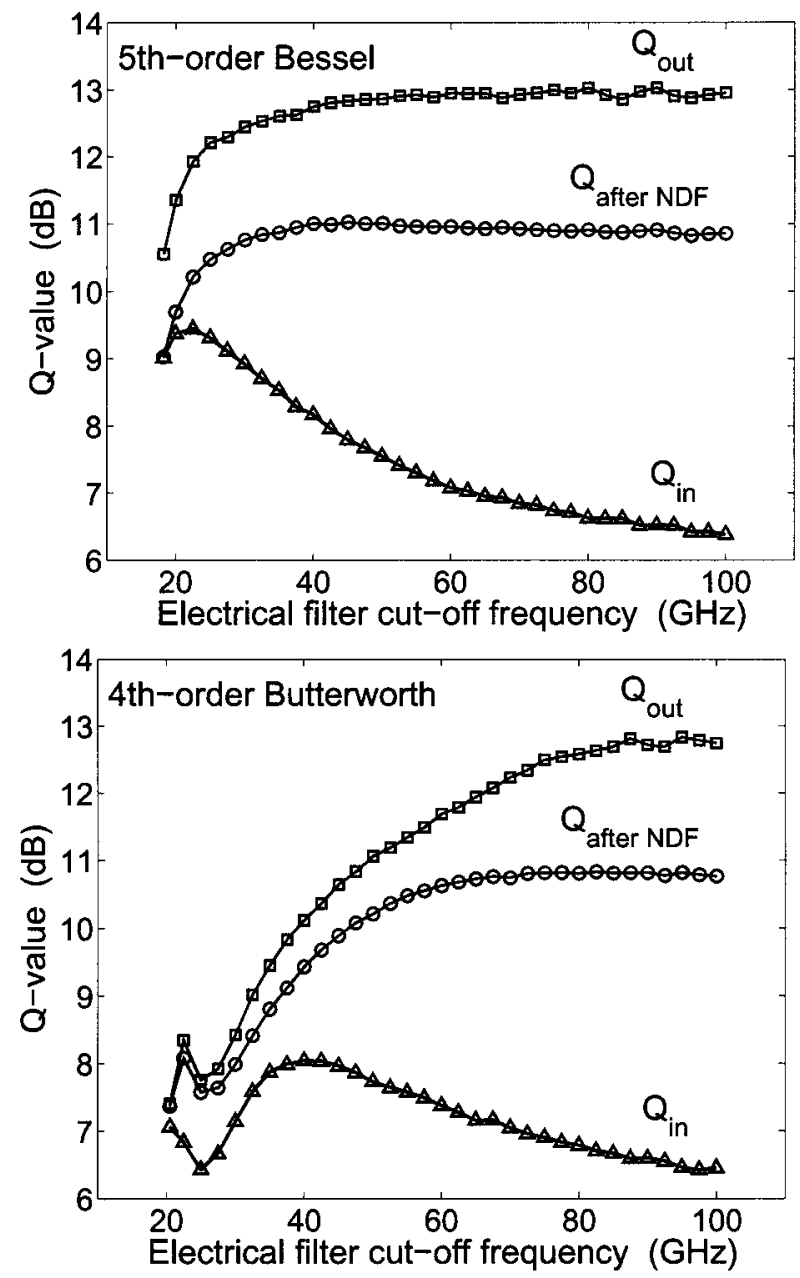

Figure 3. $Q$-values versus cut-off frequency of the receiver electrical filter.

evident from Fig. 3, where the signal $Q$-factor at the pulse processor input, $Q_{\text {in }}$, the NDF output, $Q_{\text {after NDF, }}$, and the pulse processor output, $Q_{\text {out }}$, is plotted as a function of the cut-off frequency of the receiver electrical filter for two commonly encountered filter types. Here, the EDFA gain and the NDF length are the same as those used in Fig. 2. For the conventional receiver (see $Q_{\text {in }}$-curve), when the cut-off frequency is too low, the signal quality is degraded by the increase of inter-symbol interference and the decrease of pulse amplitude. Large cut-off frequency leads to a decrease of the SNR in the detected electrical signals, and this also results in degradation of the signal quality. On the other 
hand, when the proposed method is employed, the nonlinear pulse broadening in the NDF permits to improve the signal phase margin without increasing the inter-symbol interference. The nonlinear loop mirror intensity filtering improves further the quality of the detected signals. In the proposed scheme, the required electrical bandwidth is wider than that of the conventional receiver since also the pulse spectrum spreads out. However, because of the nonlinearity involved in the process, the SNR is not decreased for a wide range of cut-off frequencies. Indeed, it is seen that the $Q$-factor at the NDF output and the pulse processor output stabilises and does not degrade any further after some value of the cut-off frequency in the considered frequency range. The asymptotic $Q$-factor at the pulse processor output is more than $3 \mathrm{~dB}$ higher (corresponding to a linear improvement factor of more than 2) than the $Q$-factor $Q_{\text {in }}$ for the conventional receiver with the optimum cut-off frequency. We mention that for larger cut-off frequencies the signal quality will be eventually degraded by the receiver thermal noise.

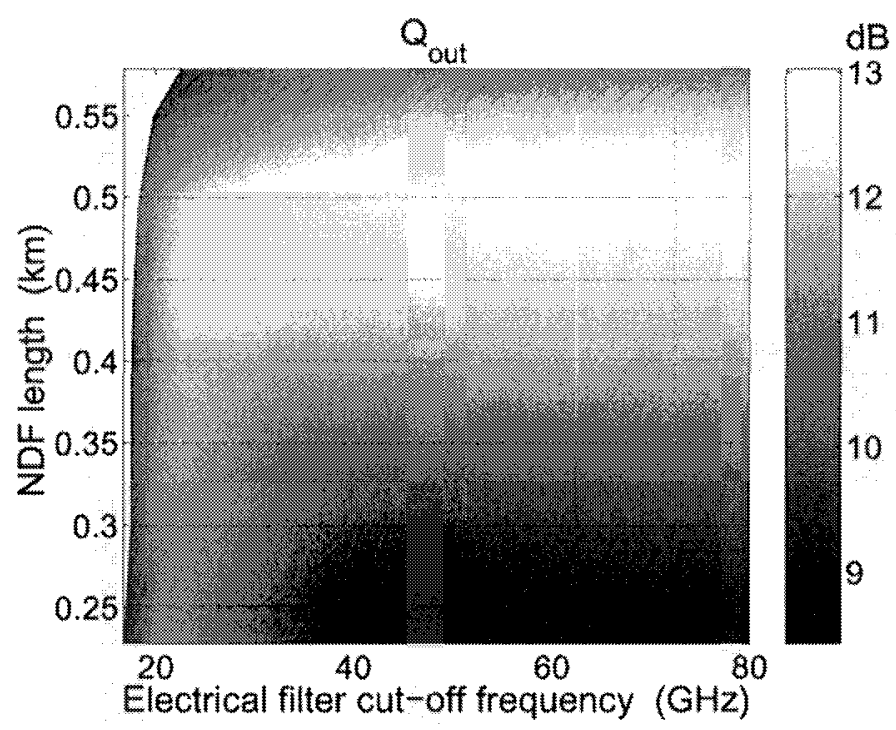

Figure 4. $Q$-factor at the pulse processor output versus receiver electrical cut-off frequency and NDF length.

A key parameter to be tuned in the proposed pulse processor is the length of the NDF. Figure 4 shows the $Q$-factor at the pulse processor output as a function of the cut-off frequency of the receiver electrical filter and the NDF length. Here, a fifth-order Bessel filter is used, and the conditions $Q_{\text {out }} \geq$ $Q_{\text {after NDF }} \geq Q_{\text {in }}$ are required to be met. For each value of the NDF length, the gain of the EDFA is adjusted so as to provide both adequate enhancement 
of the nonlinearity in the NDF and adequate power at the NOLM input. It is seen that the pulse processor works with NDF lengths between approximately 0.2 and $0.6 \mathrm{~km}$, and the optimum NDF length is $0.5 \mathrm{~km}$. The decrease of $Q_{\text {out }}$ for lengths shorter/longer than the optimum one at a fixed cut-off frequency is due to the fact that the power level of pulses at the NDF output differs from the correct power level for the NOLM to operate in the region just after the switching peak. It can also be seen that for each allowed NDF length, the $Q$-factor asymptotically stabilises at some value with increase of the cut-off frequency.

\section{SUMMARY}

We have described a novel all-optical pulse processing technique for use at a $\mathrm{RZ}$ optical receiver that exploits the intensity filtering action of a NOLM for reamplification and cleaning up of the optical signals and the Kerr effect in a NDF for improvement of the signal phase margin. The efficiency of the proposed pulse processor has been demonstrated by application to jitter-limited $40 \mathrm{Gbit} / \mathrm{s} \mathrm{RZ}$ data transmission. The estimated $Q$-factor has been improved by more than $3 \mathrm{~dB}$ compared to the conventional receiver.

\section{REFERENCES}

[1] B. Bakhshi, P. A. Andrekson, M. Karlsson, and K. Bertilsson, "Soliton interaction penalty reduction by receiver filtering," IEEE Photon. Technol. Lett., vol. 10, pp. 1042-1044, 1998.

[2] S. Boscolo, S. K. Turitsyn, and K. J. Blow, "Study of the operating regime for alloptical passive $2 R$ regeneration of dispersion-managed $R Z$ data at $40 \mathrm{Gbit} / \mathrm{s}$ using in-line NOLMs," IEEE Photon. Technol. Lett., vol. 14, pp. 30-32, 2002.

[3] S. Boscolo, S. K. Turitsyn, and K. J. Blow, "All-optical passive 2R regeneration for $N \times$ $40 \mathrm{Gbit} / \mathrm{s}$ WDM transmission using NOLM and novel filtering technique," Opt. Commun., vol. 217, pp. 227-232, 2003.

[4] M. Suzuki, H. Toda, A. H. Liang, and A. Hasegawa, "Improvement of amplitude and phase margins in an RZ optical receiver using Kerr nonlinearity in normal dispersion fiber," IEEE Photon. Technol. Lett., vol. 13, pp. 1248-1250, 2001.

[5] M. Suzuki and H. Toda, "Q-factor improvement in a jitter limited optical RZ system using nonlinearity of normal dispersion fiber placed at receiver," in Tech. Dig. OFC 2001, Anaheim, California, WH3, March 17-22, 2001.

[6] H. Nakatsuka, D. Grischkowsky, and A. C. Balant, "Nonlinear picosecond-pulse propagating through optical fibers with positive group velocity dispersion," Phys. Rev. Lett., vol. 47, pp. 910-913, 1981.

[7] N. J. Smith and N. J. Doran, "Picosecond soliton transmission using concatenated nonlinear optical loop-mirror intensity filters," J. Opt. Soc. Am. B, vol. 12, pp. 1117-1125, 1995.

[8] S. Boscolo, S. K. Turitsyn, and K. J. Blow, "All-optical passive quasi-regeneration in transoceanic $40 \mathrm{Gbit} / \mathrm{s}$ return-to-zero transmission systems with strong dispersion management," Opt. Commun., vol. 205, pp. 277-280, 2002. 